In Crescendo, 2019; 10(1): 245-275

Fecha de recepción: 27 de enero del 2019

Fecha de aceptación: 26 de marzo del 2019

\title{
COMPORTAMIENTO ORGANIZACIONAL DEL DOCENTE UNIVERSITARIO ANTE EL CAMBIO INSTITUCIONAL DE LA UNIVERSIDAD POLITÉCNICA TERRITORIAL JOSÉ ANTONIO ANZOÁTEGUI (UPTJAA)
}

\author{
ORGANIZATIONAL BEHAVIOR OF THE UNIVERSITY TEACHER \\ BEFORE THE INSTITUTIONAL CHANGE OF THE UNIVERSIDAD \\ POLITÉCNICA TERRITORIAL JOSÉ ANTONIO ANZOÁTEGUI (UPTJAA)
}

\author{
Juan José Hernández-Marcano, \\ María Lourdes Piñero-Martín ${ }^{2}$
}

\section{RESUMEN}

Se trata de un proyecto de investigación macro que tiene como objetivo configurar una aproximación teórica etnográfica del comportamiento organizacional en la Universidad Politécnica Territorial "José Antonio Anzoátegui” (UPTJAA). El artículo presenta los hallazgos parciales que orientan la intención investigativa de describir el comportamiento organizacional del colectivo social docente en dicho contexto universitario. El método utilizado fue el etnográfico hermenéutico, siendo empleadas las técnicas para la recolección de información la observación participante (diario de campo), el grupo focal y entrevistas en profundidad. A los efectos del presente artículo se presentan los resultados obtenidos mediante la técnica de la entrevista en profundidad realizada a ocho (8) “versionantes”. El diseño de investigación se orientó

1 Docente Investigador Categoría Asociado de la Universidad Politécnica Territorial "José Antonio Anzoátegui”. Doctor en Gerencia. Coordinador de la Línea de Investigación "Gerencia y Tecnología Educativa”. hernandezmjj@gmail.com Número ORCID: http://orcid.org/0000-0003-1737-5880.

2 Docente investigadora Categoría titular de la Universidad Pedagógica Experimental Libertador, Instituto Pedagógico de Barquisimeto. Doctora en Ciencias, Mención Investigación. Coordinadora de la Línea de investigación "Red de Investigación Cualitativa en Educación". Malopima11@gmail.com Número ORCID: http://www.orcid.org/0000-0002-7038-2871 
en la perspectiva metodológica cualitativa por lo que la información recolectada se sistematizó mediante el proceso de codificación y categorización de los relatos proporcionados en las entrevistas. Seguidamente se realizó una “descripción densa” o interpretativa a tres categorías principales transversales: como son: (a) Espacios introspectivos del comportamiento, (b) Actuaciones comunes y el sentido de las interrelaciones, y (c) Matices sistémicas en la dinámica comportamental. Como reflexión final se tiene que en el comportamiento de los docentes resalta el predominio excesivo de la politización y burocracia partidista, lo cual genera rechazo, aislamiento y separación entre grupos, conformado un ambiente de conformismo, de disociación institucional y de deriva gerencial e institucional hacia lo que se esperaba que este tipo de universidades impactase en el bienestar social, científico y tecnológico del territorio regionaly local.

PALABRAS CLAVE: Comportamiento organizacional, docente universitario, etnografía hermenéutica.

\begin{abstract}
A macro research project aims to configure an ethnographic theoretical approach to organizational behavior at the Universidad Politecnica Territorial "José Antonio Anzoátegui" (UPTJAA). The article presents the partial findings that guide the investigative intention of describing the organizational behavior of the social teaching collective in said university context. The method used was the ethnographic hermeneutics, using the techniques for collecting information, participant observation (field diary), focus group and in-depth interviews. For the purposes of this article, we present the results obtained through the technique of the in-depth interview carried out on eight (8) "versionantes". The research design was oriented in the qualitative methodological perspective, so the information collected was systematized through the process of coding and categorizing the stories provided in the interviews. Then a "dense description" or interpretative was made to three main transversal categories: how are: (a) Introspective spaces of behavior, (b) Common actions and the sense of interrelationships, and (c) Systemic nuances in behavioral dynamics. As a final reflection, the teachers behavior highlights the excessive predominance of politicization and partisan bureaucracy, which generates rejection, isolation and separation between groups, conforming an environment of conformism, institutional dissociation and managerial and institutional drift towards the that this type of universities was expected to have an impact on the social, scientific and technological well-being of the regional and local territory.
\end{abstract}

KEY WORDS: Organizational behavior, university teacher, hermeneutical ethnography. 


\section{INTRODUCCIÓN}

Fundamentalmente, las organizaciones como sistemas sociales deben engranar con las expectativas y metas de sus miembros y viceversa, estableciendo así una relación síncrona donde la dependencia entre estos se conjugue con la efectividad de sus acciones y así poder responder eficientemente a los objetivos planteados por las mismas, tomando en cuenta sus intereses. Es imperante considerar tal afinidad en la medida de lograr el éxito organizacional, por la cual los miembros podrán sentirse coparticipes de este logro, consecuencia directa de la cooperación y colaboración gestada entre ellos.

La relación entre la organización y sus miembros será asertiva debido al comportamiento organizacional que ha de servir como un eje transversal en el éxito de la misma, donde converjan fuerzas individuales propias de cada uno de ellos, con las fuerzas de trabajo en equipo, con el fin último de entramarse con las fuerzas propias a nivel estructural de la organización, y así emerja una sinergia positiva que permita satisfacer las necesidades individuales de estos, pero también que se puedan cumplir de manera eficaz y eficiente con las metas propias establecidas a nivel colectivo. Es por ello que ante cualquier situación de cambio o transformación que vivencia la organización, ocurren manifestaciones en la manera de conducirse de las personas que la conforman, bien sea para beneficio o bien sea para obstaculizar dicho cambio.

Ahora bien, las universidades como organizaciones en sí y más allá de su trascendente misión académico social, también están constituidas por individuos y/o grupos cuyas actuaciones apuntan a la consecución de ciertos objetivos mediante la distribución de un conjunto de funciones y actividades, racionalmente direccionadas, administradas, con cierta continuidad en el tiempo, en aras de lograr pertinencia y adaptabilidad a las constantes exigencias del entorno local y nacional, donde se circunscribe, y global por demás.

Ante este compromiso, la universidad no solo debe hacer los cambios que la afectan en lo interno, sino también responder y aportar las soluciones a las nuevas formas de actividad económica, tecnológica, social, política y cultural. Tales dimensiones subentienden los nuevos desafíos que hay que afrontar y que obligan a la universidad, además de repensar sus funciones, su misión e incluso sus finalidades, adaptarse y desarrollar las estrategias relacionadas con diversos ámbitos de impacto como: gobierno, regionalización, tecnologías de la comunicación y la información, necesidades formativas y científicas de las industrias, ecología y 
ambiente, entre otras; todo lo cual ha venido configurando una realidad del entorno cada vez más dinámico y cambiante.

De allí que las instituciones universitarias no pueden enajenarse de la realidad compleja en que vivimos, en tanto han de estar en constante movimiento, con la finalidad de poder asumir, enfrentar y adaptarse a los cambios emergentes que se suscitan en la actualidad. En pleno siglo XXI no es posible imaginar una universidad estancada e inflexible, pues requieren constituirse como las organizaciones garantes de asumir cambios del entorno desde una mirada endógena, es decir, consolidar una cultura que permita la flexibilización de sus estructuras y procedimientos, donde emerja la cohesión en cada uno de sus miembros y garantizar así, la supervivencia en este complejo y exigente planeta del conocimiento.

En toda transformación universitaria el papel de las personas y de su configuración como una comunidad de intereses redunda significativamente. Cualquier cambio sea empujado desde afuera, o desde la propia institución genera un impacto en la forma como se comportan la comunidad universitaria (autoridades, directivos, docentes, alumnos, administrativos, servicio, entre otros); pero también el desarrollo de este cambio también dependerá en mucho, de cómo las personas lo asuman, ello configura una dinámica o conducta social específica a cada institución. Es así como se generó un primer cuestionamiento: ¿Cuál es la naturaleza del comportamiento organizacional de los colectivos humanos universitarios ante situaciones de cambio institucional?

Al focalizar la mirada en Venezuela, se precisa que desde 1998, el Estado Venezolano ha venido desarrollando políticas que buscan transformar el escenario universitario, entre las que destacan la Misión Sucre y la Misión Alma Mater, cuyo objetivo es la municipalización y la territorialidad de la educación superior, bajo la premisa que representa una alternativa más accesible para la población estudiantil de ingresar al sistema universitario, deslastrándose de toda aquella filosofía en las que se encuentran enmarcadas las universidades tradicionales.

La Misión Alma Mater mediante el Decreto 6.650 del 24 de marzo de 2009, publicado en Gaceta Oficial № 39.148 del 27 de marzo 2009, surgió con el propósito de impulsar la transformación de la educación universitaria venezolana y propulsar su articulación institucional y territorial, en función de las líneas estratégicas del Proyecto Nacional Simón Bolívar, garantizando el derecho de todas y todos a una educación universitaria de calidad sin exclusiones. (Misión Alma Mater, 2009). 
Esta política universitaria tiene como finalidad generar un nuevo tejido institucional de la educación universitaria venezolana, con múltiples articulaciones que traten de desarrollar y transformar la educación universitaria en función del fortalecimiento del Poder Popular y la construcción de una sociedad socialista, siendo su mayor alcance la transformación de los Institutos Universitarios de Tecnologías y Colegios Universitarios a Universidades Politécnicas Territoriales (UPT). Las UPT son instituciones que deberán vincularse con la vocación y necesidades productivas, sociales y culturales de espacios territoriales determinados, destinadas a democratizar el acceso a la Educación Universitaria y dinamizar el desarrollo endógeno, abarcando distintos campos de conocimiento, estructurando una oferta académica flexible, capaz de ajustarse a las variaciones en las demandas de formación.

Las nacientes UPT se diferencian de las universidades tradicionales debido a que su estructura curricular se enmarca en los Programas Nacionales de Formación (PNF) vinculados estos con las necesidades de la sociedad, por lo que los estudiantes están en contacto con la práctica en las comunidades y las industrias desde el primer trayecto de sus estudios, compenetrándose con las organizaciones del Poder Popular, el desarrollo tecnológico al servicio de la Nación y los problemas de la producción de bienes y servicios, en la perspectiva del Proyecto Nacional Simón Bolívar. (Misión Alma Mater, 2009).

La génesis de las UPT está bien definida desde su accionar epistemológico, ontológico, axiológico y pedagógico, en el sentido que se convierten en pilares fundamentales para su desarrollo y permanencia, de modo que el cambio sufrido por estas instituciones debió ser guiado en términos de que se minimizará el impacto latente al cual estaban expuestas. De hecho, sus estructuras tuvieron que ser desarticuladas y reconstruidas bajo un ambiente nuevo ensombrecido por la politización reinante en el país

Ante este escenario de transformación institucional el Instituto Universitario de Tecnología "José Antonio Anzoátegui”(IUTJAA) localizado en la ciudad de El Tigre en el oriente del país, en 2014 pasó a constituirse como Universidad Politécnica Territorial "José Antonio Anzoátegui" (UPTJAA), conjuntamente con la transformación de otros 29 Institutos y Colegios Universitarios a Universidades Politécnicas Territoriales, las cuales se conciben como: "Instituciones vinculadas a la vocación y necesidades productivas, sociales y culturales de espacios territoriales determinados (ciudades, estados, distritos funcionales, provincias federales), destinadas a democratizar el acceso a la Educación Universitaria y dinamizar el desarrollo endógeno". (Misión Alma Mater, 2009;p.7). 
Uno de los investigadores de la presente investigación es miembro del personal docente de la referida Institución, siendo testigo del sin número de problemas, inconformidades y malestares, pero también de retos y emprendimientos asumidos por todos los miembros de la comunidad universitaria y especialmente por el colectivo docente ante un proceso único y sin precedentes en la historia institucional. Fue así como en las observaciones iníciales realizadas durante reuniones departamentales, eventos institucionales, conversaciones informales con directivos y colegas en los espacios de interacción cotidiana, fue posible palpar numerosos encuentros y desencuentros, que iban desde el compromiso, el entusiasmo y optimismo, hasta disposiciones de resistencia, apatía o enfado. Fue así como surgió una de las primeras inquietudes generadoras de la investigación: ¿cuáles son las percepciones, valores y creencias de los docentes respecto a su desempeño individual y colectivo en la dinámica organizativa transformacional en el contexto de la UPTJAA?

La investigación buscó configurar teoréticamente desde una perspectiva etnográfica, el entendimiento descriptivo e interpretativo del comportamiento organizacional en el contexto de la UPTJAA, resaltado en un hecho de cambio institucional. Es decir, el fenómeno objeto de estudio no es aquí el cambio organizacional en sí mismo, sino el comportamiento organizacional ante un hecho de transformación o desplazamiento institucional impulsado desde "afuera" como parte de una política estatal. Tal emprendimiento significó tomar la decisión de transitar los senderos novedosos de la heurística y de la intersubjetividad; por ello nos adentramos en la realidad organizacional desde un proceso científico de "hermenéusis" basada en la "descripción densa" sobre la que descansa el método etnográfico interpretativo.

Es así que en el presente artículo se exponen los hallazgos parciales del estudio desde una de las intenciones investigativas como fue la de describir, re-crear y comprender todas aquellas situaciones e inquietudes vividas por parte del grupo docente ante la asunción transformacional a una Universidad Politécnica Territorial.

\section{CONTEXTO REFERENCIAL TEÓRICO}

\section{Sobre el comportamiento organizacional}

Ivancevichy otros (2006) plantean el comportamiento organizacional como:

Estudio del comportamiento, actitudes y desempeño humano en un entorno organizacional; implica basarse en teorías, métodos y principios 
extraídos de disciplinas como la psicología, sociología y antropología cultural para aprender sobre las percepciones, valores, capacidades de aprendizaje y acciones individuales mientras se trabaja en grupos y dentro de la organización en su conjunto, así como analizar el efecto del ambiente externo en la organización en sus recursos humanos, misiones, objetivos y estrategias. (p.10)

De manera que el comportamiento organizacional entrelaza los componentes individuales, individual, grupal y estructural (organizacional), considerándolo así multidisciplinario, en la medida de que se aprehende de diversas disciplinas. Los mencionados autores, así otros como Robbins y Judge (2009), Chiavenato (2009), concuerdan que el comportamiento organizacional no es una disciplina, ni una ciencia generalmente aceptada con un fundamento teórico establecido, lo reseñan como un campo de estudio que se desarrolla cada vez más obteniendo un impacto significativo, dentro del ámbito gerencial.

El abordaje del comportamiento organizacional implica el dimensionamiento de tres componentes fundamentales: el individual, el colectivo y la organización propiamente dicha. En el primer componente se agrupan un cúmulo de características que empiezan desde la aptitud, la cual se relaciona directamente con los aportes que pueda generar dentro de la organización alineada con el puesto de trabajo a ejercer; las características biográficas, implica conocer en profundidad la esencia de la persona, siendo necesario contar con un expediente explícito en torno a la vida de este; y aprendizaje, aval de cambio en el comportamiento, de modo que la aplicación de estrategias de reforzamiento coadyuvan al crecimiento de la persona repercutiendo en su modo de aprender traduciéndose en su comportamiento.

Además, dentro de este componente, las actitudes de las personas son destellos que auguran situaciones, ya sean positivas como negativas, impactando directamente en sus comportamientos, lo que se religaría además con sus valores y la personalidad, debido a que, el conocimiento del sistema de valores pudiera visualizar su forma de actuar, además que la personalidad debe engranar con la filosofía de la organización, en la medida de su ajuste evitando así los conflictos.

Es por eso que, la percepción del individuo lo refiere Robbins y Judge (2009) es: "...los individuos se comportan de una manera determinada no basados en cómo es su ambiente externo, sino en cómo lo perciben o creen que es.” (p.162). De allí que la percepción que tenga la persona de su trabajo, evaluaciones, condi- 
ciones, entre otros; influye en los niveles de productividad, así como también en la satisfacción, lo cual pudiera provocar el ausentismo. De manera que, tal característica es un condicionante en el proceso de toma de decisiones que asume este dentro de la organización, explicando y prediciendo entonces su forma de comportarse.

La motivación en el individuo renace desde la fuerza interior para hacer las cosas, planteándose metas, que le permitan involucrarse con sus pares y con la organización, pero que esta le responda reconociéndolo como una persona capaz y útil, donde participe en las decisiones en las que su trabajo se vea afectado, así como también se vincule su desempeño con las recompensas obtenidas, aflorando un sentido de equidad que le genere la confianza necesaria.

Por consiguiente, las emociones y los estados de ánimo del individuo son congruentes con lo descrito, en la medida que representa la condición afectiva de los mismos, no son separables de este, ayudando así a sus niveles de autoestima y actitudes, permeando a la vez su comportamiento dentro de la organización, lo cual permitirá entonces describirlo y predecirlo también.

Ahora bien, en el componente grupal se relaciona con el desempeño grupal, el cual está condicionado con la percepción de los roles, normas, cohesión de los miembros, entre otros. Estos factores contribuyen directamente a la efectividad del grupo, pero también se vincula con la satisfacción del individuo, debido a que marcará los niveles de estrés y por ende afectará el funcionamiento del grupo.

Es necesario entonces la conformación de equipos que les permita cooperar entre ellos, realizando actividades en conjunto fluyendo efectivamente la comunicación, dejando a un lado todo interés personal, para que emerja toda contribución grupal que apunte al bienestar del mismo, en ese sentido la comunicación asume un rol protagónico, siendo esta un elemento clave en la satisfacción del individuo, su asertividad provoca reducción de niveles de incertidumbre, distorsiones, incongruencias, entre otros. Los canales de comunicaciones son esenciales para que la dirección del grupo sea la correcta, sus miembros se sentirán satisfechos al momento de sentirse escuchados, permeando así la efectividad en toda la estructura del grupo.

Asimismo, el poder y política son elementos de este plano grupal. Pues el primero que los demás miembros del grupo tengan relación de dependencia con los líderes, y el segundo porque es responsabilidad de estos que asuman la naturaleza política de la organización, lo que le permitirá predecir cuáles son las accio- 
nes que han de realizar en pro de lograr ventajas a la gestión, y así dirimir la tensión que se pueda suscitar.

Es entonces que los conflictos generalmente siempre se presentan, lo cual es un factor que en la mayoría de las veces afecta al funcionamiento del grupo, por ende el manejo de los conflictos es la estrategia a emplear, considerando aspectos como: competencia, colaboración, evita el conflicto, acomodo, compromiso, entre otros, de manera que se pueda mediar y negociar con la finalidad de resolver toda situación conflictiva que logre resolver creativamente desde la mirada de las partes involucradas.

El último componente vinculado a la organización o estructura de la organización, conforma y consolida todo el accionar de la organización, pues en esta se plantea toda la estructura formal de la misma, estableciendo las líneas de mando, niveles jerárquicos, los controles, entre otros, mismos que definirán su filosofía y por ende sus estrategias, la cual hilvanarán su propia cultura, esta será el producto de la percepción subjetiva de cada uno de sus miembros.

La cultura organizacional puede arraigarse favorable o desfavorablemente, afectando directamente los niveles de productividad de la organización, es allí que los planes individuales y grupales deben engranar para que esta así se consolide eficientemente. Gran parte de la consolidación de una cultura, recae en la política y práctica de recursos humanos propia de la organización, debido a que, el proceso de selección, los programas de capacitación y desarrollo, la evaluación de desempeño, son generadores y motores para que la misma promueva una cultura efervescente a los cambios, a la dinámica cotidiana en las que están imbuidas.

\section{EL ESCENARIO UNIVERSITARIO ANTE EL CAMBIO Y CÓMO SE COMPORTA EL UNIVERSITARIO}

La dinámica que hoy vivimos hace que nos paseemos por linderos de la incertidumbre propia del cambio que se suscita en nuestra sociedad, mismos que no son definidos de manera simple, por lo que deben abordarse desde una mirada compleja, y que obligan a los distintos ámbitos de la sociedad a enfrentarlos. Estos provienen de la muy renombrada globalización, que ha promovido un avance tecnológico vertiginoso que nos exige a repensar y a recrear nuevas estrategias para asíaprehenderlos.

Lo antes descrito nos detiene a reflexionar ante la inquietud: ¿Cómo la universidad se prepara para asumir el cambio? Una respuesta que debe ser concebida 
desde el seno de la universidad considerando además las exigencias del entorno, las universidades desde su génesis han contribuido a la formación del hombre, asumiendo cada vez más un rol protagónico dentro la sociedad porque así mismo tal sociedad se lo ha exigido, en el sentido de que esta deberá religarse a los fenómenos que emergen ya sea nivel político, económico, social, tecnológico, cultural entre otros; intentando dar respuesta ante la multiplicidad de interrogantes que se han venido gestando a través de los siglos.

Lo resaltado se corresponde con lo que expone Dridi y Crespo (1999) quienes afirman que: "el cambio es un proceso frecuentemente complejo que afecta una parte o el conjunto del sector universitario, por la modificación de diversos elementos de la estructura y de las estrategias en la toma de decisiones" (p. 12).

De modo que, el cambio bajo un panorama de complejidad e incertidumbre obliga que las universidades tengan que reestructurarse en ese dinamismo, lo que implicaría una flexibilidad de toda su estructura marginada de todo principio de estabilidad que la religue a la rigidez y que sucumba por su estancamiento.

La universidad se encuentra en una encrucijada la cual deberá tomar el camino más turbulento, presentándosele muchos senderos permeados de inquietudes, exigencias, negaciones, negociaciones, crisis, entre otros; que le permitirán así definirse ante la sociedad.

Es por ello que la universidad no puede enajenarse de las exigencias de la sociedad, esta es quien debe estar enraizada con el aparato productivo de la sociedad para que si pueda existir una relación asertiva, que se traduzca en un direccionamiento proporcional de acuerdo a la formación y preparación profesional del individuo y el impacto de este en el sector productivo, de manera que la universidad no debe ni puede encerrarse dentro sus muros, todo lo contrario tiene que accionar en las sociedades.

Por su parte, Casas (2000) señala que haciendo uso de los enfoques de la Reingeniería, se pueden crear ciertos principios que apunten las respuestas necesarias para las necesidades, entre los que se resumen los siguientes:

1. Principio Relacional: consiste en la sustitución de la concepción de un "campus" autocontenido y aislado, por una institución interrelacionada y cooperativa, entramada en redes que amplíen significativamente su campo de acción. 
2. Principio Organizacional: que la universidad se alinee bajo los parámetros de una organización apropiada y sistémica que la diferencie de la actual.

3. Principio Tecnológico: la universidad debe estar vinculada con los avances tecnológicos mundiales, para así dar la mejor solución de los problemas que repercutirá de manera asertiva en la toma de decisiones en cuanto a los aspectos estructurales, académicos, administrativos, financieros y operacionales.

4. Principio de Aprendizaje Institucional: se debe dar paso a un proceso colectivo de aprendizaje, que le permita entender la necesidad del cambio organizacional, su aporte a dicho proceso y su preparación y actualización para manejar eficazmente, tanto los diseños para el aprendizaje, como las tecnologías avanzadas que se incorporarán progresivamente.

5. Principio de Financiamiento: se debe incorporar rápidamente otras fuentes de financiamiento, que refuercen los aportes presupuestarios. (p.95)

Estos principios se entretejen con la efervescencia de los cambios, trastocando así la misión de la universidad, debido a que estos son dados en los diversos subsistemas de la sociedad, lo que implica que esta quede entramarse en los mismos incidiendo directamente en una renovación profunda de todas sus aristas, por lo que su personal no deberá estar vendado ante tal realidad.

El universitario entendido en esta disertación como un docente, quien asumirá un papel trascendental que ayudará así a la universidad a trascender los cambios, en el sentido de que este alineará sus funciones académicas, de investigación y de extensión bajo una mirada profunda y transformadora que se enmarque en los principios de la continuidad que le permita adoptar una visión de una realidad ilimitada, dinámica y compleja que converja así a las necesidades de la sociedad.

Estos tendrán que replantearse desde su accionar haciendo mención desde la influencia de las tecnologías en el aparato productivo hasta los problemas socio económicos, políticos, culturales, entre otros que aquejan a las comunidades; es un despertar de la conciencia de que forma parte de una cultura "multifenoménica" que cada vez más se entrelaza dando origen a nuevos fenómenos que no pueden ser aislados.

Esta obligación a una nueva conciencia por parte del universitario apunta a patrones de comportamiento que hacen redibujar sus funciones dentro de la uni- 
versidad y por ende en la sociedad, planteando así nuevas formas de interrelación que dan pie al surgimiento de estructuras formales e informales como un agente catalizador para la asunción de los cambios.

Por lo que, es vital que el universitario se abra ante una realidad desconocida e incierta y pueda así izar banderas hacia nuevos pensamientos donde la innovación se convierta en una herramienta que permita sobrellevar la complejidad emergente continua, y que su integración con sus pares se convierta en enriquecidas bases sólidas que soporten asertivamente las nuevas formas de relacionarse en pro de lograr la sustentabilidad de la universidad en las venideras décadas.

\section{MÉTODOS}

\section{POSTURA PARADIGMÁTICA}

Según Piñero, Rivera y Esteban (2018) la coherencia paradigmática de un estudio apunta hacia el esclarecimiento y permanente vigilancia epistemológica que el investigador asume y mantiene durante el ejercicio investigativo respecto a los postulados y principios filosóficos que le dan sentido al enfoque epistémico, al paradigma, al método y a la perspectiva metodológica en la cual transitará.

El enfoque epistemológico vincula los marcos referenciales de pensamiento de hacer ciencia y refiere según Padrón (2014) "un sistema profundo de convicciones acerca de qué es la Ciencia, la Investigación y el Conocimiento Científico, acerca de cuáles son las vías más eficientes para producir y legitimar el conocimiento científico, acerca de las fuentes y operaciones del conocimiento científico" (p.3). De acuerdo a las distinciones presentadas por este autor, los enfoques epistemológicos son: Empirista, Racionalista y Viviencialista.

De allí que a los efectos de la presente investigación asumimos este último enfoque, en tanto no se trata de descubrir o inventar un conocimiento dado por la razón, sino que se trata de un acto de comprensión haciendo énfasis en la noción de sujeto y de realidad subjetiva, por encima de la noción de objeto o de realidad objetiva, en tanto se tiene la convicción de que la vía más apropiada para acceder al conocimiento es una simbiosis entre el sujeto investigador y su objeto de estudio. Es decir, una especie de identificación sujeto-objeto tal, que el objeto pase a ser una experiencia vivida, sentida y compartida por el investigador (de ahí el calificativo de Vivencial). Por eso el acercamiento al objeto de estudio resultó de una concepción ontológica del saber "producto del conocimiento y las interpretaciones de los simbolismos socioculturales a través de los cuales los actores de un 
determinado grupo social abordan la realidad (humana y social, fundamentalmente)." (Padrón, 2014; p.04).

Asimismo, según Padrón (2014) los paradigmas refieren "re-encarnaciones" de los enfoques epistemológicos enunciados por grupos de poder por mantener una "ciencia normal" en la defensa de ciertos patrones de rigor en el hacer investigativos; de allí que a estas corrientes se les reconoce por el sufijo "ismos". Dentro del abanico de distinciones de paradigmas que existen en el mundo de la ciencia, los investigadores de este estudio se guiaron por los principios del "interpretativismo".

Ergo, Guba y Lincoln (1994) caracterizan a este paradigma bajo una ontología realista donde la misma emerge de las construcciones sociales, enraizada a una epistemología subjetiva que no busca diferenciar entre el sujeto y el objeto de conocimiento, donde sus principios metodológicos son posibles desde una mirada que converja la heurística y dialéctica, con una profunda reflexión hermenéutica, con el fin de entramar una realidad social donde no se busque explicar, traducir ninguna teoría, sino que se comprenda la misma desde la percepción y vivencia en que sucede por las personas relacionadas.

\section{RESULTADOS}

\section{SOBRE EL MÉTODO ETNOGRÁFICO INTERPRETATIVO}

La elección del método, se inclinó por la tradición etnográfica-hermenéutica o etnografía interpretativa postulada por Clifford Geertz, quien refiere que el contexto cultural está estructurado con mitos, símbolos y creencias, los cuales constituyen aspectos que configuran la cosmovisión e identificación que facilita la interpretación, y esta a su vez, es obtenida con la descripción densa.

La distinción de que la etnografía es "descripción densa" refiere una idea general que busca estructuras de significación presentes en el ambiente cultural que se estudia, para analizar la interacción y qué consecuencias tiene para los que viven y actúan. La interpretación se presenta en este caso como el modo o acción de "Interesarse en el mundo", en el que la realidad cobre sentido y significado para el individuo. Desde esta perspectiva, para Geertz (1992) "la descripción etnográfica presenta tres rasgos característicos: es interpretativa, lo que interpreta es el flujo del discurso social y la interpretación consiste en tratar de rescatar 'lo dicho' en ese discurso de sus ocasiones perecederas y fijarlo en términos susceptibles de consulta" (p.32). 
Para la ejecución del método que guió el proceso de investigación, se asumió la propuesta de Velasco y Díaz (2006), quienes resaltan tres (3) fases que se distinguen como: Acciones, Objetos y Transformaciones.

Tabla 1

FASES DEL MÉTODO DE ESTUDIO

\begin{tabular}{c|ll}
\hline Fases del Método & \multicolumn{2}{|c}{ Descripción de las acciones implícitas desarrolladas } \\
\hline \multirow{4}{*}{ Acciones } & a) & Concepción del Proyecto. \\
\hline & b) & Abrir un diario de campo. \\
\hline & C) & Elección de campo. \\
\hline & d) & Entrada al campo. \\
\hline & e) & Adopción de Roles y asimilación de rutinas. \\
\hline & f) & Fin del trabajo de campo. \\
\hline \multirow{4}{*}{ Objetos } & a) & Situarme en el interior del laberinto investigativo. \\
\hline & b) & La ausencia de la dirección. \\
\hline \multirow{4}{*}{ Transformaciones } & c) & Los objetos cobran diferentes perfiles y perspectivas \\
\hline & & (amalgamas). \\
\hline & a) & De la presencia a la interacción e información. \\
\hline & b) & De la interacción y la información al registro. \\
\hline & c) & Del registro al contenido. \\
\hline & d) & Del contenido al texto. \\
\hline & e) & Interpretación del texto. \\
\hline
\end{tabular}

Fuente: elaboración propia.

\section{DISEÑO DE LA INVESTIGACIÓN}

A efectos de la presente investigación se asumió la consideración de la perspectiva metodológica cualitativa, entendida por Piñero, Rivera y Esteban (2018) como la dinámica interactiva de construcción y reconstrucción de estrategias técnico-operativas realizadas por el investigador (o investigadores), en el marco del método científico elegido y guiado mediante la reflexión epistémica constante que implica el involucramiento en el contexto social, a los fines de recrear, analizar y comprender o transformar las significaciones cotidianas del fenómeno en estudio por parte de los informantes participantes.

Por su parte Salinas (2009) señalan que la perspectiva cualitativa implica, en primer lugar, un estilo de investigación social en el que se da una insistencia especial en la recogida esmerada de datos y observaciones lentas, prolongadas y sistemáticas en base a notas, ejemplos, grabaciones, entre otras. Todo ello supuso en la realización de la investigación asumir un diseño abierto, flexible y recursivo en el uso de estrategias y multi técnicas para la recolección y análisis de la información. En este artículo presentaremos los hallazgos devenidos de la entrevista en profundidad. 
Ahora bien, se hizo imprescindible la elección de informantes (aquí llamados "versionantes"), considerados como parte del personal docente ordinario o contratado con experiencia superior a los cinco (5) años de antigüedad, con categorías académicas que abarcan desde instructor, asistente, agregado, asociado y titular, conformando un total de ocho (8) docentes para la fase de entrevistas, a los cuales se les identifico con la nomenclatura: V1, V2, V3...V8. Con respecto a la técnica de recolección de información Taylor y Bogdan (1994), definen la entrevista en profundidad como los encuentros cara a cara entre el entrevistado y los informantes, encuentros que son dirigidos hacia la comprensión de las perspectivas que tienen los informantes respecto a sus vidas, experiencias o situaciones, tal como lo expresa con sus propias palabras; la entrevistas en profundidad siguen un modelo de conversación entre iguales.

Para la entrevista se utilizó un guion no estructurado con inquietudes claves de planteamientos generadores, apoyados con una libreta de notas, lápiz y grabadora digital de audio, procesados con el programa de edición de Sound Forge versión 7.0 antes de la transcripción con el procesador de textos. Esta técnica e instrumentos posibilitaron alcanzar un estilo relacional que enfatizó en la valoración contextual social e interacción de sentidos y significados entre los actores sociales y con respecto a ellos, convirtiéndose en un espacio para intersubjetividad.

Luego de la transcripción de las entrevistas en los protocolos debidamente identificados por versionante, se procedió a la codificación y categorización del texto, como paso clave en el proceso de interpretación o "hermenéusis". Tanto la codificación como la categorización procuran esfuerzos sistematizadores de la información que se traducen en un trabajo de conceptualizar, clasificar y relacionar, en donde el investigador aporta su propia interpretación de la realidad objeto de estudio, siendo lo referido por Giddens (2001) cuando indica que a la hermenéutica del sujeto, el investigador debe sumarle su propia hermenéutica y es en esta relación doble donde aparece la interpretación y comprensión de la realidad estudiada.

Cabe destacar que en el proceso de sistematización de la información se realizó en primera instancia siguiendo la codificación abierta, y luego de varios espacios de reflexión, revisión y contrastación, se realiza la codificación axial. Es en esta fase donde se van agrupando las frases de los versionantes por subcategoría y luego por dimensiones. Sin embargo, por razones de espacio en el artículo presentaremos las frases agrupadas en forma general por subcategorías, realizando una selección de las más llamativas: 


\section{HERMENEUSIS DE LOS HALLAZGOS}

La importancia de las prácticas sociales cotidianas del colectivo docente dentro de la UPTJAA, definen su dinámica "comportamental" dentro de esta, es decir, cómo los procesos individuales que conforman la esencia del docente, impactan e influyen en las relaciones interpersonales, grupales y organizacionales. En ese sentido, develar el comportamiento organizacional del docente en la UPTJAA en el marco de su transformación institucional, implicó

Tabla 2

INTEGRACIÓN DE CATEGORÍAS (ENTREVISTAS)

\begin{tabular}{|c|c|c|}
\hline \multicolumn{3}{|c|}{$\begin{array}{l}\text { Categoría Medular: } \\
\text { Comportamiento Organizacional del docente en la UPTJAA }\end{array}$} \\
\hline \multicolumn{3}{|c|}{ Entrevista en profundidad } \\
\hline Categoría Principal & Subcategoría & Dimensión \\
\hline \multirow{2}{*}{$\begin{array}{l}\text { Espacios introspectivos del } \\
\text { comportamiento personal }\end{array}$} & Rasgos de Personalidad & $\begin{array}{l}\text { Valores } \\
\text { Percepciones personales } \\
\text { Motivación personal } \\
\text { Aprendizaje Individual }\end{array}$ \\
\hline & $\begin{array}{l}\text { Ejercicio académico desempeñado } \\
\text { en la UPTJAA }\end{array}$ & $\begin{array}{l}\text { Reconocimiento del compromiso hacia el } \\
\text { ejercicio docente } \\
\text { Satisfacción hacia el trabajo }\end{array}$ \\
\hline \multirow{2}{*}{$\begin{array}{c}\text { Actuaciones comunes y el sentido } \\
\text { de las interrelaciones }\end{array}$} & Vinculación con la gerencia & $\begin{array}{l}\text { Liderazgo } \\
\text { Relación de poder - política }\end{array}$ \\
\hline & Vinculación con los pares docentes & $\begin{array}{l}\text { Comunicación } \\
\text { Pertinencia institucional de la actuación } \\
\text { colectiva } \\
\text { Valores de trabajo en grupo }\end{array}$ \\
\hline \multirow{3}{*}{$\begin{array}{l}\text { Matices sistémicas } \\
\text { organizacionales }\end{array}$} & $\begin{array}{l}\text { Vinculación con los principios de la } \\
\qquad \text { UPT }\end{array}$ & $\begin{array}{l}\text { Respecto a la Estructura y diseño de la } \\
\text { organización: } \\
\text { Respecto a los principios rectores de las } \\
\text { UPT. }\end{array}$ \\
\hline & $\begin{array}{c}\text { Vinculación con la Gestión } \\
\text { Académica } \\
\text { UPT }\end{array}$ & $\begin{array}{l}\text { Creación del conocimiento } \\
\text { Selección del personal docente }\end{array}$ \\
\hline & $\begin{array}{l}\text { Actuaciones del docente y su } \\
\text { interrelación con el buen ejercicio } \\
\text { organizacional }\end{array}$ & $\begin{array}{l}\text { Adaptación al cambio } \\
\text { Politización en las relaciones } \\
\text { Alineación y sinergia organizacional }\end{array}$ \\
\hline
\end{tabular}

Fuente: elaboración propia. 
conocer desde las voces de los informantes, y las observaciones especificas e inespecíficas registradas durante el transitar investigativo, cómo el docente definido como un ser único, social, cultural, político, actúa dentro de los espacios de la universidad, así como también su interrelación y vinculación con sus pares, y además cómo se comporta ante las características propias de la estructura universitaria.

En la tabla 2 es apreciable la significación de la categoría medular configurada como: comportamiento organizacional del docente en la UPTJAA y tres (3) categorías principales con subsiguientes subcategorías y dimensiones.

\section{HeRmeneusis de la CATEgoría Principal: Espacios INTROSPeCtI- VOS DEL COMPORTAMIENTO PERSONAL}

La categoría principal: "Espacios introspectivos del comportamiento personal" intentó conocer al colectivo docente desde su esencia como un ser permeado de actitudes, percepciones, sentimientos, valores, que definen su personalidad, capacidad de aprendizaje, entre otros.

De modo que la personalidad enmarcada en aquellas características y rasgos que responsabilizan el comportamiento del docente ante situaciones, edificadas bajo principios éticos y morales que moldean los valores, motivación y las actitudes, cristalizando el hecho de percibir causas y consecuencias que se suscitan del vivir; convirtiéndose así en cimientos que consolide el aprendizaje individual como un atributo transversal de la personalidad.

Y es que las personas se convierten en piedra angular para el desarrollo exitoso de cualquier organización, indistintamente al ramo al que se dediquen, simplemente estas forman parte del entramado organizacional, al cual se ajustarán a una misión y visión que de soporte a la consecución asertiva de las metas planteadas. En ese sentido, cobran protagonismo las personas, ya sea desde su desenvolvimiento personal así como también grupal. Desde la perspectiva individual la personalidad de los miembros de la organización es factor crucial para su éxito, pues según Allport (citado por Rubio y Castro, 2012):

Cada persona posee unas características diferenciadoras respecto a otras, definiendo su forma de comportarse, agregando que la personalidad es como una organización dinámica de los sistemas psicofísicos únicos de cada individuo, los cuales llegan a determinar su adaptación al medio, incluyendo el ámbito laboral. (p.03) 
Las aptitudes dentro del comportamiento individual del docente se enlazaron con la Trayectoria Profesional y Ejercicio académico desempeñado dentro de la Institución, partiendo de un amplio bagaje académico que les permite desempeñarse en otras instituciones y a diferentes niveles, además de la aplicación de estrategias genuinas dentro de las unidades curriculares administradas. Por lo que las aptitudes apuntan a la generación de conocimiento dentro su radio de acción, abriendo el espectro de entendimiento al momento de relacionarse con los demás docentes, es decir, le servirá para el análisis y diagnostica ante situaciones complejas.

En la apreciación del comportamiento del colectivo docente surge la percepción de cómo se ven ellos mismos, y cómo consideran que los perciben sus compañeros, lo que implica la manera de cómo ellos son percibidos ante el resto de los demás, develándose una imagen subjetiva de su esencia personal. Por otra parte, la satisfacción en el trabajo, forma un aspecto individual de cómo el docente se siente identificado dentro de la Universidad, para esto él diseña la forma de trabajo dentro de sus espacios, lo que configura su ejercicio académico realizado en la UPTJAA.

Los docentes de la UPTJAA se ven asimismo con valores que transitan la ética, la responsabilidad, respeto, colaboración y honestidad, esto los hace percibirse como personas proactivas, emprendedoras, comprometidas, lo cual se constituye en anclajes interesantes para motivarse a ser personas pacíficas, identificadas con el cambio, que buscan prepararse cada día en preparase y superarse como profesionales, para que ello signifique en un impacto positivo en la labor cotidiana en el aula con sus estudiantes. Tales percepciones personales son un reflejo importante de cómo pueden comportarse como individuos ante cualquier situación de cambio o transformación institucional, pues cuando se trata de personalidad, a decir de Robbins y Jugde (2009): "estas características ya están definidas cuando un individuo se integra a la fuerza de trabajo, y en su mayor parte hay poco que la gerencia pueda hacer para modificarlas." (p.32). Sin embargo, más allá de cómo se perciben a sí mismos de forma individual, será necesario también, cómo esto pudiera reflejar los comportamientos compartidos e interrelacionados en los espacios sociales, dialógicos, de liderazgo y gerenciales que conforman la universidad.

A continuación en la tabla 3 se aprecian algunas voces de los versionantes, las cuales por razones de espacio hemos procurado incluir las más significativas: 
Tabla 3

\section{SISTEMATIZACIÓN DE LAS VOCES DE LOS VERSIONANTES PARA LA CATEGORÍA ESPACIOS INTROSPECTIVOS DEL COMPORTAMIENTO PERSONAL SEGÚN ENTREVISTAS EN PROFUNDIDAD}

\begin{tabular}{|c|c|c|}
\hline Subcategoría & Dimensiones & Las voces de los versionantes \\
\hline $\begin{array}{c}\text { Rasgos de } \\
\text { Personalidad }\end{array}$ & $\begin{array}{l}\text { Valores } \\
\text { Percepciones } \\
\text { personales } \\
\text { Motivación } \\
\text { personal } \\
\text { Aprendizaje } \\
\text { Individual }\end{array}$ & 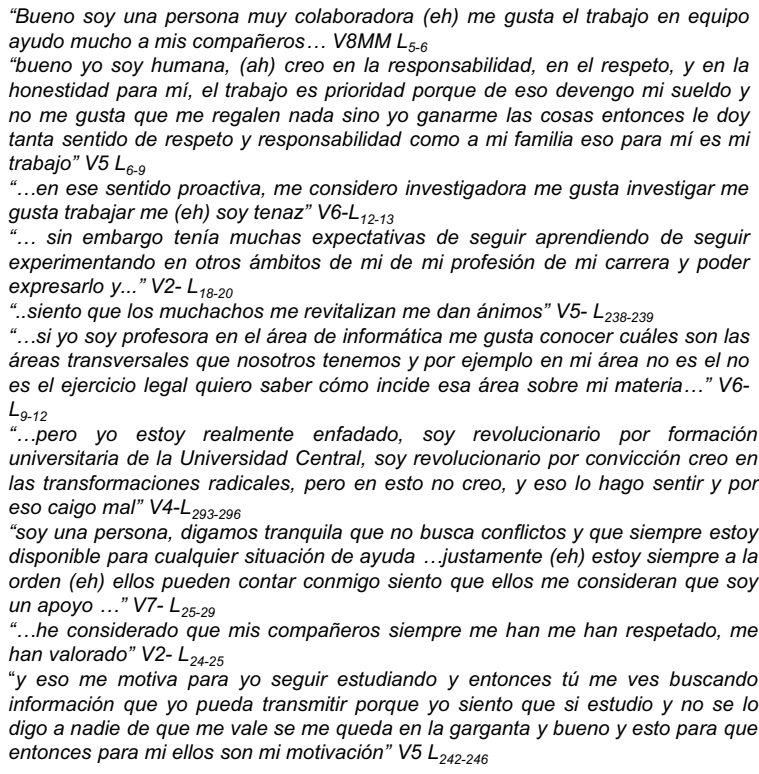 \\
\hline $\begin{array}{c}\text { Ejercicio } \\
\text { académico } \\
\text { desempeñado } \\
\text { en la UPTJAA }\end{array}$ & $\begin{array}{l}\text { Reconocimiento } \\
\text { del } \\
\text { compromiso } \\
\text { hacia el } \\
\text { ejercicio } \\
\text { docente } \\
\text { Satisfacción } \\
\text { hacia el } \\
\text { trabajo }\end{array}$ & $\begin{array}{l}\text { “... a nivel pues a nivel profesional me siento muy identificado porque puedo } \\
\text { ejercer mi profesión" V4- } \mathrm{L}_{99-100} \\
\text { "por lo tanto yo procuro diariamente actualizarme, yo estudio todos los días sobre } \\
\text { problemas tipo sanitarios y mi responsabilidad con los muchachos es enseñarle } \\
\text { todo lo técnico que yo pueda en función del programa que tiene la materia" V5- } \\
\mathrm{L}_{33-42} \\
\text { "...yo hago revolución en el aula pero revolución del pensamiento, que el } \\
\text { ciudadano sepa a que vino, para que estudia, y que es lo que va lograr, ese } \\
\text { joven, ese alumno, ese dicente, entiende que realmente él está aquí para } \\
\text { formarse,..." V4- } \mathrm{L}_{254-257} \\
\text { porque el trabajo educativo no es un trabajo individual, es un trabajo } \\
\text { mancomunado, y por lo tanto se necesita de diferentes elementos para que se de } \\
\text { ese proceso (eh) no depende solamente de mi, pero al yo poner ese granito de } \\
\text { arena (eh) colaboro a que el proceso sea dinamizador sea proactivo y pues se } \\
\text { logre todo lo que se espera a nivel educativo y a nivel académico" V7- } \mathrm{L}_{30-39}\end{array}$ \\
\hline
\end{tabular}

Fuente: elaboración propia. 


\section{Hermeneusis de la CATegoría Principal: Actuaciones COMUNES Y EL SENTIDO DE LAS INTERRELACIONES}

La importancia de los grupos dentro de las organizaciones asume su relevancia en la medida que tratan de moldear el comportamiento de cada uno de sus miembros. Indistintamente su filosofía está formada por grupos, en la mayoría heterogéneos, que buscan satisfacer sus necesidades, desde una óptica individual, pero a su vez grupal con el fin último de alinearse a las metas y objetivos propuestos por dichas organizaciones.

En ese sentido, los grupos han de conformarse bajo principios sinérgicos que contribuyan al desarrollo de acciones conducentes efectivas relacionadas con los planes y objetivos de la organización. Estos siempre tienen una meta en común, y por esa están dispuestas a trabajar cada uno de sus integrantes, partiendo de sus necesidades individuales engranándose con los intereses del grupo.

La categoría principal: "Actuaciones comunes y el sentido de las interrelaciones" buscó develar las interacciones entre el colectivo docente, considerando cómo el liderazgo ejerce sobre los grupos docentes, así como también la conformación de equipos de trabajos se alinea a los procesos de comunicación asertiva, generando esquemas de satisfacción y confianza, con la intención de manejar efectivamente los conflictos en pro de las relaciones de poder y política que se suscitan.

El desenvolvimiento grupal por parte de los miembros de una organización responde principalmente a la forma como se interrelacionan, en función al cumplimiento de las metas de la misma, pero además de satisfacer las propias. Por lo que han de seguir una serie de normas y patrones que condicionan su comportamiento al momento de relacionarse, tales normas responderán a los intereses y funciones de cada organización, y es por eso que hace única y diferente a las organizaciones, en la medida de que estas cobran vida por su grado de madurez en su forma de interrelacionarse.

En la vinculación de los miembros de una organización emergen múltiples factores, tales como: equipos de trabajo, comunicación, conflictos, entre otros, en virtud de lo afirmado por Chiavenato (2009) al plantear que dentro las características fundamentales para la cohesión de los grupos en las organizaciones, deben estar motivados al trabajo en equipo, con la finalidad de percibirse como una unidad donde coexistan coincidencias y desacuerdos entre sus miembros, de modo que bajo ese ambiente se condiciona el comportamiento del grupo dentro de dichas organizaciones. 
Al focalizar la mirada en los relatos de los versionantes es posible interpretar que si bien es cierto existe una interrelación generalizada de amabilidad, respecto y disposición al trabajo en equipo, devenida del reconocimiento del comportamiento individual colaborativo, en las actuaciones en colectivo se dibuja un comportamiento de resistencia, desmotivación y apatía sobre todo del personal con cierto tiempo en la institución hacia el personal de nuevo ingreso. Este escenario se debe fundamentalmente al rechazo hacia el cambio institucional a universidad politécnica territorial por parte de un importante sector del colectivo docente, en tanto lo relacionan con el desplazamiento de procesos académicoadministrativos llevados a cabo de manera autónoma a una inherencia del factor de "politización" en la toma de decisiones importante; de allí que el liderazgo

Tabla 4

\section{SISTEMATIZACIÓN DE LAS VOCES DE LOS VERSIONANTES PARA LA CATEGORÍA ACTUACIONES COMUNES Y EL SENTIDO DE LAS INTERRELACIONES}

\begin{tabular}{|c|c|c|}
\hline Subcategoría & Dimensiones & Las voces de los versionantes \\
\hline $\begin{array}{l}\text { Vinculación con } \\
\text { la gerencia }\end{array}$ & $\begin{array}{l}\text { Liderazgo } \\
\text { Relación de } \\
\text { poder - política }\end{array}$ & 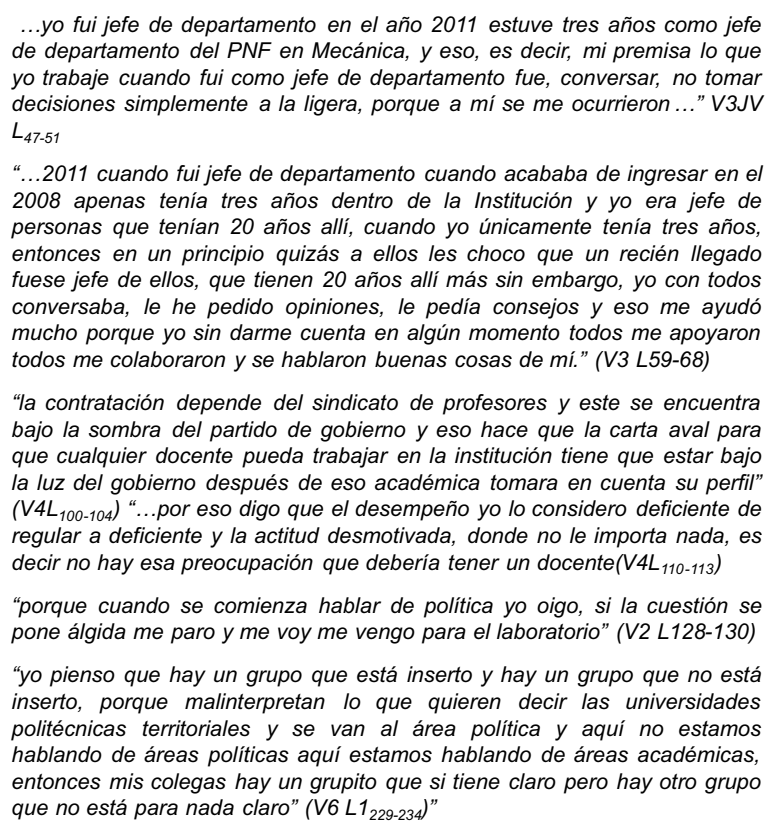 \\
\hline
\end{tabular}




\section{Tabla 4 (Cont.)}

\begin{tabular}{|c|c|c|}
\hline Subcategoría & Dimensiones & Las voces de los versionantes \\
\hline $\begin{array}{l}\text { Vinculación con } \\
\text { los pares } \\
\text { docentes }\end{array}$ & $\begin{array}{l}\text { Pertinencia } \\
\text { institucional de } \\
\text { la actuación } \\
\text { colectiva }\end{array}$ & 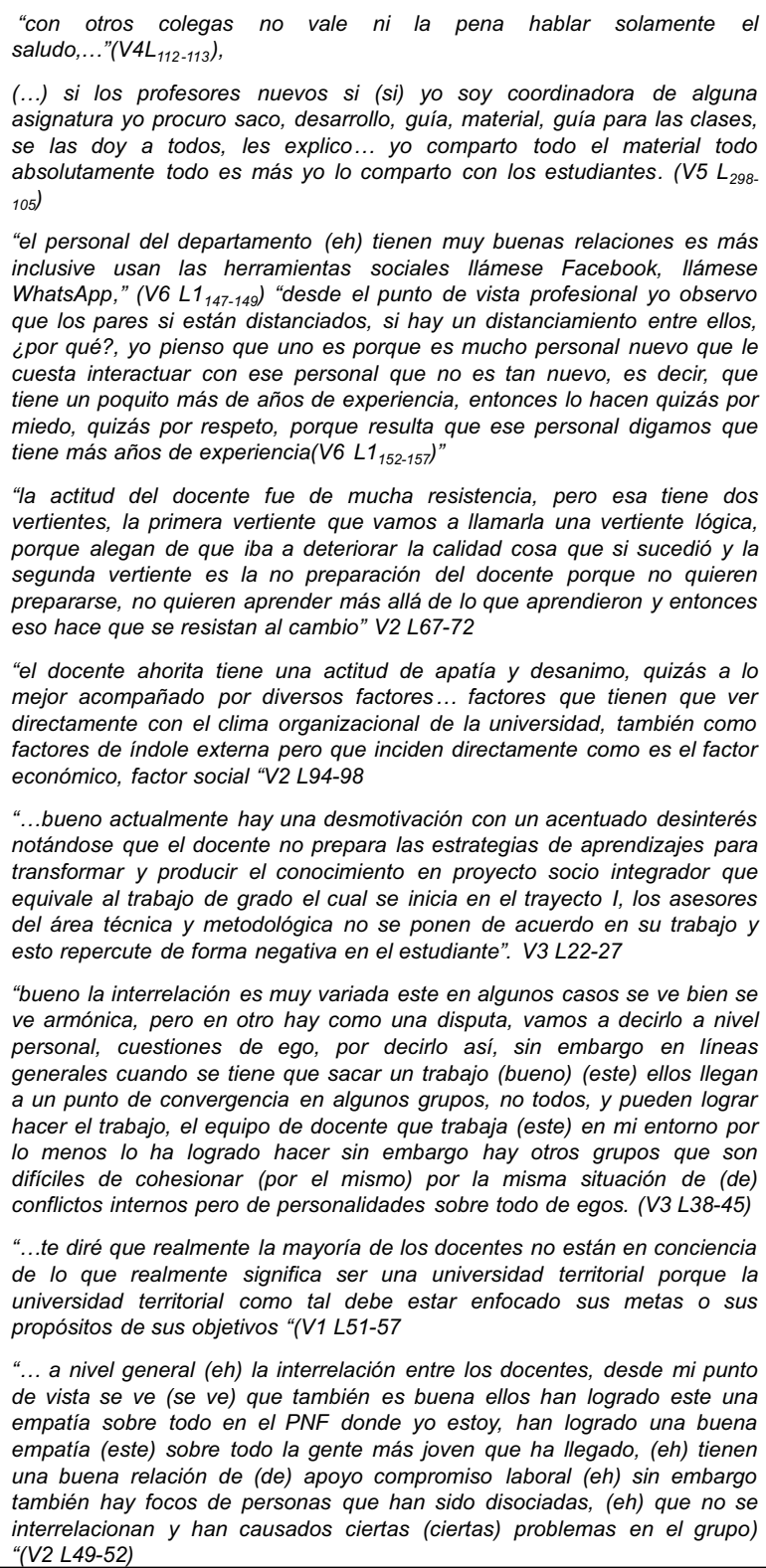 \\
\hline
\end{tabular}

Fuente: elaboración propia. 
existente está vinculado a disposiciones verticales gubernamentales, y no existe una toma de decisiones horizontal y deliberada de acuerdo a las necesidades y circunstancias propias de la institución.

Cabe señalar también que la politización dentro de la UPTJAA ha hecho estragos en la relación de los docentes, colocando por encima la ideología partidista en detrimento de los valores humanos, sociales, afectivos y académicos, a tal punto de resquebrajarse una relación profesional signada de vivencias en la Institución por décadas, por la sencilla razón de pertenecer a partidos políticos opuestos. Esto ha generado divisiones, poca cohesión social, rechazos y muy bajo compromiso con los nuevos lineamientos organizacionales que corresponden y se han establecido a una universidad politécnica territorial.

La tabla 4 presenta algunas de las frases rescatadas de los entrevistados.

\section{HERMENÉUsis de la CATEgoría PRINCIPAL: MATICES SISTÉMiCAS ORGANIZACIONALES}

Es necesario partir de la premisa que las relaciones entre los individuos y los grupos dentro de las organizaciones direccionan su accionar comportamental, y a su vez crean una cultura en donde converjan intereses propios y colectivos, generando así una idiosincrasia organizacional. Es por ello que, las organizaciones deben ser consideradas como organismos sociales vivos, en la que miembros ejercerán roles de liderazgos, pero otros serán seguidores, conformando así una dupla recíproca que garantice el éxito de la organización.

La categoría principal: "Matices sistémicas en la dinámica comportamental", supuso develar la esencia de la UPTJAA en términos de su estructura propiamente definida, con una cultura organizacional que la caracteriza, además de las políticas y prácticas, la cual impacta en la actuación de los grupos docentes e igualmente en el docente en sí. En ese sentido, la estructura y diseño de la universidad se alineó a la génesis de las Universidades Politécnicas en marco de la territorialidad, la cual buscó conocer como la UPTJAA impactó en las comunidades en función a sus necesidades y exigencias, relacionando la actuación del docente ante la asunción de la territorialidad en su praxis académica.

Dentro de las políticas y prácticas gestadas en la UPTJAA, se intentó entramar aspectos gerenciales que trastocaron la dinámica de la antigua institucionalidad, impactando directamente en el colectivo docente, debido a que condiciona 
en cierta parte su comportamiento porque se vincula con los mecanismos de selección de personal, de supervisión, la gestión académica avizorada en términos de expansión de la Institución, vinculada además con las funciones académicas bajo la sombra de la territorialidad.

Como se aprecia en el cuadro 5, en las voces de los entrevistados se interpreta que las actuaciones comunes y sus interrelaciones colectivas expresadas en la categoría principal anterior, se vinculan con el sentido sistémico de desapego a los nuevos principios que rigen la nueva UPT luego de más de 5 años de su implementación, en tanto por un lado los docentes no ven los resultados prácticos y de pertinencia con el entorno territorial, al tiempo que se aprecia una apatía y desconocimiento hacia los documentos que sustentan tales principios. Tal desconocimiento a su vez no permite que la actuación en el aula (desde el punto de vista de planificación y curricular) del docente esté separada de los lineamientos que establece el gobierno para este tipo de universidades.

Igualmente, los nuevos requerimientos de nuevo ingreso del personal docente, no siguen mecanismos de selección y capacitación adecuados que permitan no solo prepararlos y orientarlos para el cumplimiento de sus funciones docentes, en virtud que muchos de ellos son universitarios sin componente pedagógico. Asimismo, la apatía, la desmotivación y la resistencia a estos nuevos lineamientos generan en los docentes a una "inmovilización" hacia los procesos creadores de conocimiento mediante la investigación, toda vez que sobre esta función recae la presencia académica de una universidad.

Es significativo destacar que hay un reconocimiento de adaptación al cambio de algunos docentes, pero sin la convicción plena del éxito y pertinencia de esta institucionalidad, en tanto se aprecia una añoranza a la cultura y formas de apego a la antigua institucionalidad como instituto tecnológico. Los docentes resaltan el predominio excesivo de la politización y burocracia partidista, lo cual genera rechazo, aislamiento y separación entre grupos, conformado un ambiente de conformismo, de disociación institucional y de deriva gerencial e institucional hacia lo que se esperaba que este tipo de universidades impactase en el bienestar social, científico y tecnológico del territorio regional y local. 
Tabla 5

SISTEMATIZACIÓN DE LAS VOCES DE LOS VERSIONANTES PARA LA
CATEGORÍA MATICES SISTÉMICAS ORGANIZACIONALES

\begin{tabular}{|c|c|c|}
\hline Subcategoría & Dimensiones & Las voces de los versionantes \\
\hline $\begin{array}{c}\text { Vinculación } \\
\text { con los } \\
\text { principios de la } \\
\text { UPT }\end{array}$ & $\begin{array}{l}\text { Respecto a la } \\
\text { Estructura y } \\
\text { diseño de la } \\
\text { organización: } \\
\text { Respecto a los } \\
\text { principios } \\
\text { rectores de las } \\
\text { UPT. }\end{array}$ & 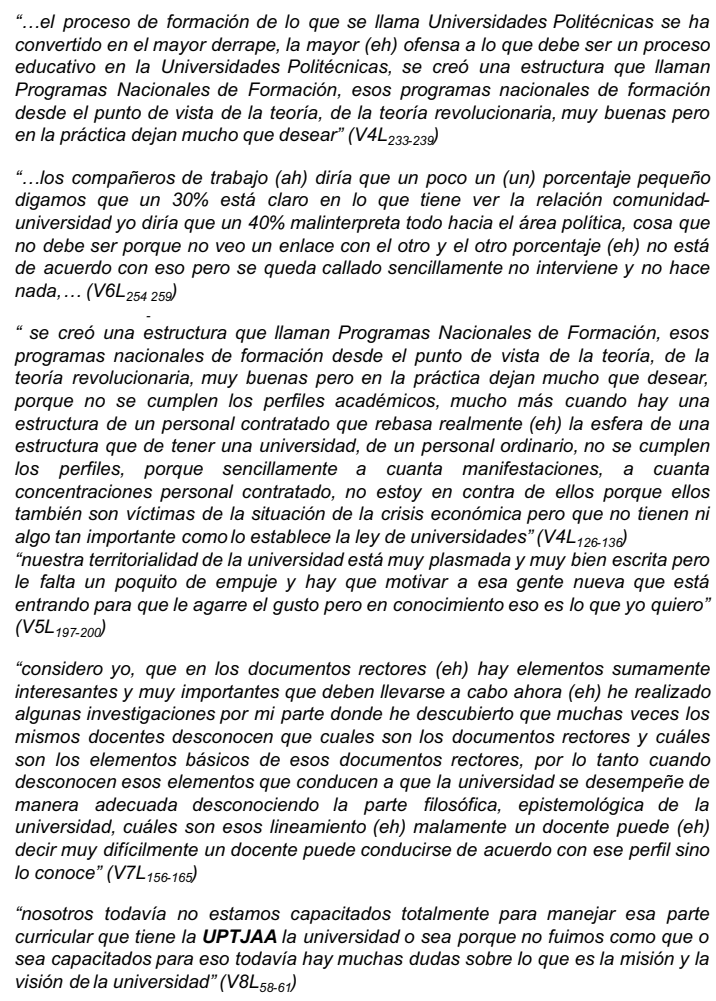 \\
\hline $\begin{array}{c}\text { Vinculación con } \\
\text { la Gestión } \\
\text { Académica } \\
\text { UPT }\end{array}$ & $\begin{array}{l}\text { Creación del } \\
\text { conocimiento }\end{array}$ & $\begin{array}{l}\text { "... en esta universidad no hay investigación porque no hay ni libros, no hay ni libros } \\
\text { mucho menos, redes sociales para uno sentarse a investigar un laboratorio de } \\
\text { investigación, quienes somos el personal de dedicación exclusiva que debemos } \\
\text { emplear horas para la investigación o redactar folletos libros escritos manuscritos } \\
\text { nada no hay nada entonces (eh) esto implica que esos docentes también inmersos } \\
\text { en esta mediocridad en esta mediocridad de docentes..."V4LG } L_{337-343} \\
\text { (...) porque los demás no están haciendo investigaciones, lo que están haciendo son } \\
\text { proyectos de grado a nivel de pregrado pero no trabajo de investigación ..." V6TH } \\
L_{389-406} \\
\text { "... la universidad no tiene el mecanismo para hacer esa selección de ese personal, } \\
\text { sino sencillamente ella se limite este es ingeniero en sistema este es, este aquel, } \\
\text { este es el otro, y te encasilla..." (V6 } L_{121-123)} \\
\text { "entonces tengo una materia técnica reducida a un trimestre y ahorita que unas } \\
\text { semanas muy cortas para dar un programa tan ambicioso que no me da tiempo y de } \\
\text { verdad no hay tiempo me da lástima que los muchachos a veces me quedo fuera del } \\
\text { tiempo ahorita no vengo en las tardes porque no tengo carro" (V5 } L_{82-86)}\end{array}$ \\
\hline
\end{tabular}


Tabla 5 (Cont.)

\begin{tabular}{|c|c|c|}
\hline Subcategoría & Dimensiones & Las voces de los versionantes \\
\hline $\begin{array}{l}\text { Actuaciones del } \\
\text { docente y su } \\
\text { interrelación con } \\
\text { el buen ejercicio } \\
\text { organizacional }\end{array}$ & $\begin{array}{l}\text { Adaptación al } \\
\text { cambio } \\
\text { Politización en las } \\
\text { relaciones } \\
\text { Alineación y } \\
\text { sinergia } \\
\text { organizacional }\end{array}$ & 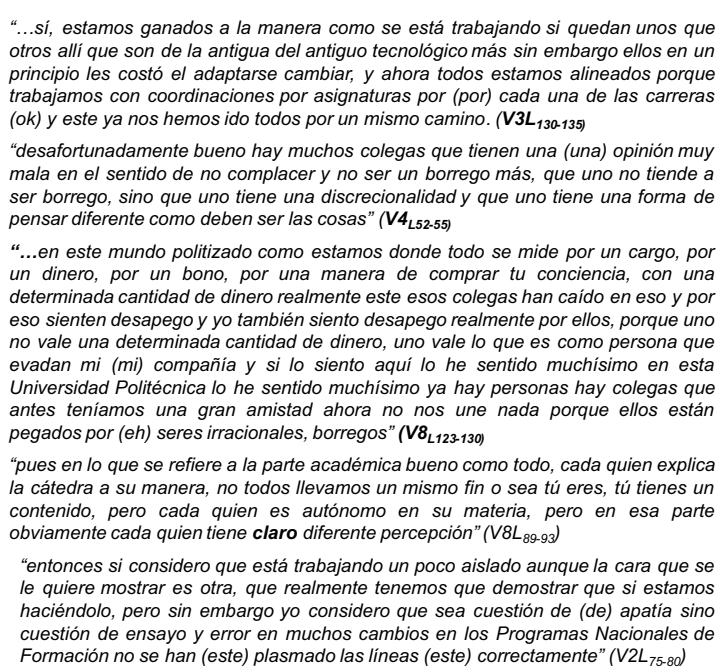 \\
\hline
\end{tabular}

Fuente: elaboración propia.

\section{DISCUSIÓN}

Según la Ley de Universidades venezolana aún vigente (1970), en su artículo 1: "La Universidad es fundamentalmente una comunidad de intereses espirituales que reúne a profesores y estudiantes en la tarea de buscar la verdad y afianzar los valores trascendentales del hombre" (p.01), además el artículo 2 menciona que estas son: "Instituciones al servicio de la Nación y a ellas corresponde colaborar en la orientación de la vida del país mediante su contribución doctrinaria en el esclarecimiento de los problemas nacionales." (p.01). Es pues la universidad la institución por excelencia donde se perfilan los muchos de los objetivos de desarrollo y bienestar social de una sociedad.

Como parte de una reorientación de políticas universitarias, el Estado venezolano promovió a partir del año 2008 una serie de transformaciones institucionales universitarias, enraizada en la masificación de la educación universitaria a través de la creación de la misión Alma Mater y Misión Sucre, consideradas complementarias en el sentido de que la primera apunta a la transformación de los Institutos Universitarios de Tecnología a Universidades Politécnicas Territoria- 
les, y la segunda resalta la municipalización de la educación universitaria. Ello significó cambios relevantes no solo en la manera de conducción gerencial, sino que los colectivos humanos que conformaban estas instituciones se sometieron a una transformación de un pensamiento académico fuertemente politizado y anclado en principios revolucionarios emanados directamente del gobierno central, por lo que no vivenciaron la autonomía que prevalecía en las universidades con más tradición en el país.

El Instituto Universitario de Tecnología "José Antonio Anzoátegui" (IUTJAA) localizado en la ciudad de El Tigre en el oriente del país, fue una de las organizaciones universitarias que pasó a constituirse como Universidad Politécnica Territorial "José Antonio Anzoátegui" (UPTJAA). Siendo uno de los investigadores del presente estudio, miembro del personal docente de dicha institución, vivenció el tránsito de este desplazamiento institucional, por lo que se emprendió una investigación que procurase describir de una manera científica cualitativa el reconocimiento del comportamiento del colectivo docente ante dicho proceso de cambio, sobre la base que toda transformación organizacional inevitablemente trastoca a las personas que la conforma y su manera de conducirse en ella.

En ese sentido, las personas mantienen una relación de reciprocidad con las organizaciones, en la medida que cada una depende de la otra para alcanzar sus propios objetivos, podría decirse que esta simbiosis es directamente proporcional, es decir, a mayor cumplimiento de metas de las personas, mayor será el éxito de dichas organizaciones, resaltando además la presencia latente del dinamismo en las que interactúan ambas partes. Pues son las personas quienes le dan vida a las organizaciones, sea cual sea su naturaleza, dado que pueden o no conducirlas al éxito dependiendo su desempeño, moldean e impulsan las acciones a seguir para garantizar la sostenibilidad, tal cual afirma Chiavenato (2009) las personas dentro de las organizaciones como: “... son el principio, el punto medio y el final de toda organización.” (p.183). Afirmación que evidencia que las organizaciones no solamente son estructuras físicas, legales y administrativas, todo lo contrario, son organismos vivos que se alimentan de cada una de las personas que están dentro de ellas, siendo únicas y diferentes.

Definitivamente, lograr el cambio en una organización tan compleja como una universidad no es una tarea fácil de hacer y de alcanzar, de allí que su implementación debe estar guiado por un proceso de equilibrio y transformación paulatina y participativa, de modo que se desarrolle de manera eficiente y convin- 
cente para que los individuos o miembros de la organización lo acepten de manera cooperativa y voluntaria. Ello implicaría gestionar procesos de planeación, diseño y evaluación de una estrategia pertinente del cambio para evitar el nacimiento de la resistencia al mismo.

Al recurrir a la realización de las entrevistas en profundidad a un variado grupo de ocho (8) docentes se pudo interpretar que desde el punto de vista individual, los colegas se ven a sí mismos como personas responsables, con valores éticos hacia el trabajo, comprometidos en sus tareas individuales, pacíficos y poco prestos a los conflictos organizacionales, así como dispuestos a prepararse profesionalmente. Esto es sumamente importante, en virtud que permite configurar una identidad del docente de la UPTJAA con valores y actitudes hacia la vida y hacia la organización muy favorables', sintiéndose satisfechos y orgullosos de haber pertenecido a la universidad en su antigua y nueva institucionalidad. No obstante, el cambio organizacional, fue impulsado por fuerzas externas, que implicó una alta politización, la apertura a nuevas formas de conducción académica, así como la incorporación de nuevo personal docente.

Pese a ello, se percibe en sus expresiones verbales una baja satisfacción respecto a este proceso, sobre todo cuando se refieren a las relaciones interpersonales que apunten a la conformación de una identidad colectiva necesaria para la facilitación del cambio. De hecho, se perciben aislados y poco cohesionados hacia la realización de tareas colectivas o grupales, con problemas de comunicación y rechazo hacia las formas de ingreso del nuevo personal docente y poco dispuestos a involucrase en el conocimiento de los documentos que sustentan los principios de la nueva institucionalización. A este respecto, no parece existir por parte de la gerencia de la UPTJAA la implementación de mecanismos de capacitación, sensibilización, seguimiento y motivación entre el personal docente para lograr una identificación con los fines de esta transformación, en virtud que esta institución, al igual que otros tantos institutos tecnológicos y colegios universitarios transitaron este cambio como parte de una política gubernamental a nivel nacional.

Es justamente este aspecto de toma de decisiones politizada por parte de un gobierno central que está ubicado en otra zona geográfica del país, respecto a la localización oriental de la UPTJAA, que ha generado resistencia, rechazo, apatía y aislamiento entre el colectivo docente, lo que nos permite cuestionarnos sobre el verdadero impacto y pertinencia de la transformación institucional llevada a cabo, en tanto los docentes no se ven a sí mismos, ni en colectivo, ni en su rela- 
ción con la organización en un estado de bienestar, equilibrio y satisfacción organizacional, respecto a los objetivos y aspiraciones de constitución en una universidad politécnica territorial.

Existe un amplio espectro de recomendaciones y clasificaciones que orientan la teoría del comportamiento organizacional, para lograr el éxito ante el cambio organizacional. Sin embargo, no podemos negar el hecho que cada organización es única, y en definitiva según el aporte de la teoría sistémica las organizaciones pueden ser conceptualizadas como entes vivos, "es una unión de partes o componentes conectados en una forma organizada" (Van Gigch, 1989), con una historia y una cultura propia. De modo pues, que cada organización, y en este caso la UPTJAA es un sistema dinámico en el que las personas que las conforman se manejan y comportan con valores, creencias, liderazgos, roles, principios, actitudes e interacciones diferenciadas de otra institución universitaria.

Consideramos que la UPTJAA asumió el reto de transformación universitaria que se imponía, sin embargo el comportamiento organizacional que ha devenido en el colectivo docente no se ha constituido en un agente potenciador y favorecedor del éxito de la nueva institucionalidad. Definitivamente no podemos ser el colectivo docente de "antes" de la transformación, porque sencillamente los docentes ya no somos los mismos. Más sin embargo, es un hecho que el ser humano visibilizado en sus docentes, secretarias, obreros, directivos y estudiantes, son el "mayor activo" de nuestra institución, por lo que aspiramos que los hallazgos parciales de esta investigación permitan a la gerencia universitaria tomar las decisiones adecuadas promuevan la flexibilidad en los procesos y en las estructuras para la adecuación del comportamiento organizacional, hacia el logro de la excelencia y la más elevada misión que como universidad tenemos ante la sociedad.

Los cambios organizacionales, dentro de lo que es el escenario de la época en que vivimos, deben entenderse como los retos permanentes capaces de asegurar el fracaso o éxito de una organización, de allí que algunas estrategias y posibilidades de avance han sido generadas en la segunda parte de esta investigación, mediante la realización de grupos focales y grupos de discusión con el personal de la universidad, pues (...) "El dinamismo con que se desarrolla la realidad actual, impone la necesidad de la adecuación continua que implica el uso de estrategias de intervención, en otras palabras: hacer algo para lograr la adaptabilidad al cambio" (Escudero, Delfín y Arano;2014,p.8). 


\section{REFERENCIAS BIBLIOGRÁFICAS}

Casas, M. (2000). Reestructuración de la universidad venezolana pública en la encrucijada. Revista Educación Superior y Sociedad. Vol. 11. Nro.: 1. Disponible en: http://ess.iesalc. unesco.org.ve/ess3/index.php/ess/article/view/357 [Recuperado: 2018, abril 21]

Chiavenato, I. (2009). Comportamiento Organizacional: La dinámica del éxito en las organizaciones. Segunda Edición. Editorial McGraw- Hill Interamericana

Dridi, H., y Crespo, M. (1999). Las actuales tendencias de cambio en las universidades frente al siglo XXI. Revista Educación Superior y Sociedad. Vol. 10. Nro.2. Disponible en: http://ess.iesalc.unesco.org.ve/ess3/index.php/ ess/article/view/344[Recuperado: 2018, mayo 10]

Escudero, J.; Delfín, L. y Arano, R. (2014). El desarrollo organizacional y la resistencia al cambio en las organizaciones. RevistaCiencia administrativa, No. 1. Disponible:

https:/www.uv.mx/iiesca/files/2014/09/01CA201401.pdf [Recuperado: 2018, junio, 25].

Geertz, C. (1992). La interpretación de las culturas. Barcelona: Gedisa.

Giddens, A. (2001) Las nuevas reglas del método sociológico. Crítica positiva de las sociologías comprensivas. Buenos Aires, Amorrortu Editores.

Guba, E. G. y Lincoln, Y. S. (1994): Competing paradigms in qualitative research, en N. K. Denzin \& Y. Lincoln (eds.): Handbook of qualitative research, Thousand Oaks, California: Sage.

Ivancevich, J., Konopaske, R., y Matteson, M. (2006). Comportamiento Organizacional. Séptima Edición. McGraw-Hill Interamericana Editores, S.A.

Ley de Universidades con su Reglamento (1970). Gaceta Oficial de la República Bolivariana de Venezuela, 28.262 (Extraordinario). Septiembre 8, 1970

Misión Alma Mater, (2009). Misión Alma Mater. Educación Bolivariana Socialista. [Documento en Línea]. Disponible en: http://www.unearte.edu.ve/media/informacion/pdf/ mision_alma_mater.pdf[Recuperado: 2018, mayo 20]

Padrón, J. (2014). Notas Sobre Enfoques Epistemológicos, Estilos de Pensamiento y Paradigmas. Proyecto de Epistemología en DVD. Doctorado en Ciencias Humanas. LUZ. [Documento en Línea] Disponible: http://padron.entretemas.com/Notas_EP-EnfEpistPdigmas.pdf. [Recuperado: 2018, mayo 16].

Piñero, M.L., Rivera, M.E. y Esteban, E. (2018) El proceder del investigador cualitativo. Precisiones para el proceso de investigación. Barquisimeto, Venezuela. UPEL-IPB.

Robbins S., y Judge, T. (2009). Comportamiento Organizacional. Decimotercera Edición. Editorial Pearson Educación. México.

Rubio, N., y Castro, M. (2012). La personalidad: variable individual y grupal en la gerencia de organizaciones. Revista CICAG. Vol.: 10. Nro.: 1. Disponible en: https://dialnet.unirioja.es/descarga/articulo/5028144.pdf [Recuperado: 2018, abril 27]

Salinas, P. (2009) La investigación cualitativa. En: Salinas, P. y Cárdenas. (2009) Métodos de Investigación social.2da Edición. Quito, Ecuador. Editorial QUIPUS CIESPAL. 
Taylor, S., y Bodgan, R. (1994). Introducción a los métodos cualitativos de investigación. Ediciones Paidos Ibérica. Barcelona - España.

Van Gigch (1989). Teoría General de Sistemas. Editorial Trillas, México.

Velasco, H., y Díaz, A. (2006). La lógica de investigación etnográfica. Quinta Edición. Editorial Trotto S.A. Madrid - España. 